\title{
Supermigrativity of aggregation functions
}

\author{
Fabrizio Durante ${ }^{\mathrm{a}, *}$, Roberto Ghiselli Ricci ${ }^{\mathrm{b}}$ \\ ${ }^{a}$ Dipartimento di Scienze dell'Economia, Università del Salento, Lecce, Italy \\ ${ }^{b}$ Dipartimento di Economia e Management, Università degli Studi di Ferrara, Ferrara, Italy
}

\begin{abstract}
A functional inequality, called supermigrativity, was recently introduced for bivariate semicopulas and applied in various problems arising in the study of aging properties of stochastic systems. Here, we revisit this notion and extend it to the case of aggregation functions in higher dimensions. In particular, we show how supermigrativity can be expressed via monotonicity of a function with respect to logarithmic majorization ordering of real vectors. Various alternative characterizations of supermigrativity are illustrated, together with some of its weaker versions. Several examples show similarities and differences between the bivariate and the general case.

Keywords: Aggregation functions, Copulas, Functional Inequalities, Multicriteria decision making, Supermigrativity.
\end{abstract}

\section{Introduction}

When the main interest is to describe and predict a system with $d$ components, it is often convenient to represent its behavior in the language of probability theory by assuming the existence of a random vector $\mathbf{X}=\left(X_{1}, \ldots, X_{d}\right)$, defined on a suitable probability space, such that $X_{i}$ may interpret the uncertainty of the $i$-th system component.

In many cases, the study of a random vector $\mathbf{X}$ can be carried out by representing its probability joint distribution function $F_{\mathbf{X}}(\mathbf{x})=\mathbb{P}\left(X_{1} \leq x_{1}, \ldots, X_{d} \leq x_{d}\right)$ as a composition of the marginal distributions $F_{1}, \ldots, F_{d}$ and a copula $C$, via the formula $F_{\mathbf{X}}=C\left(F_{1}, \ldots, F_{d}\right)$

\footnotetext{
${ }^{*}$ Corresponding author.

Email addresses: fabrizio.durante@unisalento.it (Fabrizio Durante), ghsrrt@unife.it (Roberto Ghiselli Ricci)
} 
due to the celebrated Sklar's Theorem (see, e.g., [17, 24, 29, 31]). Moreover, it is also of interest to study the aging properties of $\mathbf{X}$, namely those properties that can help interpreting the evolution of the system at different future times (see, e.g., [28, 34]).

Such studies are related to various real situations. For instance, one can interpret $\mathbf{X}$ as the vector of lifetimes of the components of an engineering disposal and, hence, the aging properties serve to indicate possible strategies in presence of the wear-out of the system. In another context, $\mathbf{X}$ can be related to lifetimes of individuals (linked, for instance, in a partnership) and the behaviour of the system in time may help in the pricing of joint life insurance policies.

While univariate notions of aging are by-now classical in the literature, when dealing with the analysis of dependent lifetimes, analogous definitions are rather controversial. A seminal contribution was provided in [5], where the non-trivial interactions between dependence properties (as described by the copula) and the aging properties were considered. Furthermore, this latter work highlighted that:

- a possible framework to study aging properties of a vector of lifetimes, in particular in the exchangeable case, is the class of semi-copulas (see, e.g., $[14,16,35]$ ), which are (fuzzy) connectives that generalize both copulas and triangular norms and have also been used in several fuzzy integrals (see, e.g., [26, 27]);

- notions of multivariate aging can be expressed in terms of functional inequalities among semi-copulas (see, e.g., $[4,5,11]$ ), which have been also developed in various related problems (see, for instance, [10, 20, 21]).

Here we focus on the so-called supermigrativity, whose definition is given below. In the following, we denote by $\mathbb{I}$ the real unit interval $[0,1]$.

Definition 1.1. A function $F: \mathbb{I}^{2} \rightarrow \mathbb{I}$ is called supermigrative if, and only if

- it is symmetric, i.e. $F(x, y)=F(y, x)$ for every $(x, y) \in \mathbb{I}^{2}$;

- it satisfies the inequality

$$
F(\alpha x, y) \geq F(x, \alpha y)
$$


for all $\alpha \in \mathbb{I}$ and for all $x, y \in \mathbb{I}$ such that $y \leq x$.

When the inequality (1) is strict for any $\alpha \in] 0,1[$ and for all $0<y<x$, we refer to strict supermigrativity.

The term "supermigrative" was used in [12] to underline the connection of this inequality with the concept of migrativity of triangular norms ( $\mathrm{t}$-norms, for short), originally formulated to study the preservation of associativity under convex combinations [15] and, hence, extended in different situations (see $[7,9,18,19]$ ). The study of supermigrativity in various classes of bivariate functions was considered in $[12,13]$. Since then, several investigations in reliability theory have underlined further applications of this concept in the comparison of random vectors (see, for instance, $[6,32,36]$ ).

Here, we aim at revisiting some results about supermigrativity for bivariate semicopulas (section 2) and present the supermigrativity of more general classes of aggregation functions by discussing similarities and differences. Then, we extend the notion of supermigrativity to arbitrary dimensions (section 3) and present some related inequalities that may arise in a natural way when one is interested in providing bounds for the aggregation process once some of the input values are multiplied by a given rescaling factor (section 4).

\section{Supermigrativity of binary semi-copulas and aggregation functions}

In this section, we will devote the symbol $S$ to an arbitrary 2 -semi-copula, i.e. a binary aggregation function with neutral element 1 (see, e.g., [17]). Note that every semi-copula $S$ satisfies $S(x, 0)=S(0, x)=0$ for every $x \in \mathbb{I}$ and, hence, Eq. (1) can be considered only for $\alpha \in] 0,1[$.

Given a supermigrative semi-copula $S$, it follows from Eq. (1), with $x=1$, that $S$ pointwise dominates the product t-norm (in symbols, $S \geq \Pi_{2}$, where $\Pi_{d}(\mathbf{x})=\Pi_{d}\left(x_{1}, x_{2}, \ldots, x_{d}\right)=$ $x_{1} \cdot x_{2} \cdots x_{d}$ for any $d \in \mathbb{N}$ ). By borrowing a terminology from copula theory (see, for instance, [17]), a binary aggregation function $A$ is said to be PQD (respectively, NQD) when $A \geq \Pi_{2}$ (respectively, $A \leq \Pi_{2}$ ). Thus, any supermigrative semi-copula is PQD. 
Remark 2.1. We emphasize that, unlike supermigrative semi-copulas, a supermigrative aggregation function need not be PQD. Consider, for instance, the aggregation function given by $A(x, y)=x y^{2}$, if $y \leq x$, while $A(x, y)=x^{2} y$, otherwise. Thus, $A$ is strictly supermigrative and, at the same time, $A(x, y)<\Pi_{2}(x, y)$ for all $\left.x, y \in\right] 0,1[$.

The supermigrativity of Definition 1.1 can be reformulated in various equivalent ways, as indicated in [12, Proposition 2.7] (see also [30]). First, for any dimension $d \geq 2$ we set $\Delta_{d}:=\left\{\mathbf{x} \in \mathbb{I}^{d}: 0<x_{d} \leq x_{d-1} \leq \cdots \leq x_{1}\right\}$.

Definition 2.1. Let $\mathbf{x}, \mathbf{y} \in \mathbb{I}^{d}$. We say that $\mathbf{x}$ is logarithmically majorized by $\mathbf{y}$ (in symbols, $\mathbf{x} \prec_{L} \mathbf{y}$ ) if, and only if, $\mathbf{x}, \mathbf{y} \in \Delta_{d}$ and the following conditions hold:

$$
\left\{\begin{array}{l}
\Pi_{k}\left(T_{k}(\mathbf{x})\right) \leq \Pi_{k}\left(T_{k}(\mathbf{y})\right) \quad \text { for all } k=1, \ldots, d-1 ; \\
\Pi_{d}\left(T_{d}(\mathbf{x})\right)=\Pi_{d}\left(T_{d}(\mathbf{y})\right)
\end{array}\right.
$$

where $T_{k}(\mathbf{x})$ is the truncated vector given by the first $k$ components of $\mathbf{x}$, with $T_{d}(\mathbf{x})=\mathbf{x}$.

Let us introduce the notion of Schur geometrical concavity (see, for instance, [33]).

Definition 2.2. A function $F: \mathbb{I}^{d} \rightarrow \mathbb{I}$ is called Schur geometrically concave if, and only if, the following conditions hold:

- it is symmetric, i.e. $F(\mathbf{x})=F\left(\mathbf{x}_{\pi}\right)$ for all $\mathbf{x} \in \mathbb{I}^{d}$ and for all permutations $\pi$ on $\{1,2, \ldots, d\}$, where $\mathbf{x}_{\pi}:=\left(x_{\pi(1)}, \ldots, x_{\pi(d)}\right)$;

- it satisfies the inequality $F(\mathbf{x}) \geq F(\mathbf{y})$ whenever $\mathbf{x} \prec_{L} \mathbf{y}$.

In particular, for $d=2$, the following result follows.

Theorem 2.1 (see Proposition 1 in [13]). A (bivariate) semi-copula is supermigrative if, and only if, it is Schur geometrically concave.

In particular, as a consequence of [23, Theorem 1.6], the following result holds. 
Corollary 2.2. Let $S$ be a symmetric 2-semi-copula that admits continuous first-order partial derivatives on $] 0,1\left[^{2}\right.$. Then $S$ is supermigrative if, and only if, for all $(x, y) \in \Delta_{2}$

$$
x \partial_{x} S(x, y)-y \partial_{y} S(x, y) \leq 0
$$

Next lemma will be useful in the sequel.

Lemma 2.3 (see Corollary 2.8 in [12]). A symmetric semi-copula $S$ is supermigrative if, and only if, $S(\mathbf{x}) \geq S(\mathbf{y})$ whenever $\mathbf{x}, \mathbf{y} \in \Delta_{2}$ satisfy the conditions $x_{1} \leq y_{1}$ and $\Pi_{2}(\mathbf{x}) \geq \Pi_{2}(\mathbf{y})$.

Remark 2.2. Notice that Theorem 2.1 and Lemma 2.3 still hold if we consider aggregation functions instead of semi-copulas.

Now, for the class $\mathscr{S}_{\text {SM }}$ of supermigrative bivariate semi-copulas some facts are easily proved:

- $\mathscr{S}_{\mathrm{SM}}$ is a closed set in the class of semi-copulas, i.e. pointwise limit of supermigrative semi-copulas (if it exists) is supermigrative;

- $\mathscr{S}_{\mathrm{SM}}$ is a convex set, i.e. for every $\beta \in \mathbb{I}$ and $S_{1}, S_{2} \in \mathscr{S}_{\mathrm{SM}}, \beta S_{1}+(1-\beta) S_{2} \in \mathscr{S}_{\mathrm{SM}}$.

- The pointwise infimum of $\mathscr{S}_{\mathrm{SM}}$ is $\Pi_{2}$, while the pointwise supremum of $\mathscr{S}_{\mathrm{SM}}$ is the minimum $\mathrm{t}-$ norm $M_{2}(x, y)=\min \{x, y\}$. In general, supremum and infimum of two supermigrative semi-copulas is supermigrative.

Although supermigrativity was implicitly introduced for continuous functions (in fact, the semi-copulas considered in [5] need to be continuous), it is easy to show that supermigrative non-continuous semi-copulas do exist.

Example 2.1. Let $S$ be the symmetric semi-copula given, for $y \leq x$, by

$$
S(x, y)= \begin{cases}x^{\beta} y, & x<\gamma, \\ \min \{x, y\}, & x \geq \gamma\end{cases}
$$

where $\beta>0$ and $\gamma \in] 0,1]$. It is quite easy to see that: 
- $S$ is both supermigrative and continuous for $\beta \leq 1$ and $\gamma=1$;

- $S$ is continuous but not supermigrative for $\beta>1$ and $\gamma=1$;

- $S$ is supermigrative but not continuous for $\beta \leq 1$ and $\gamma<1$;

- $S$ is neither supermigrative nor continuous for $\beta>1$ and $\gamma<1$.

In order to enrich our knowledge of the class of supermigrative semi-copulas, it could be of interest to check whether supermigrativity is preserved by special transformations.

We start by considering distortions of semi-copulas (see, e.g., [17]). We recall that, if $h$ is an increasing bijection of $\mathbb{I}$, then the distortion of a semi-copula $S$ is the semi-copula $S_{h}(x, y)=h^{-1}(S(h(x), h(y)))$.

However, it should be noticed that supermigrativity is not preserved by distortions. In fact, strict Archimedean t-norms are obtained as distortions of the supermigrative semi-copula $\Pi_{2}$, but there are strict and continuous Archimedean t-norms that are not supermigrative (consider, for instance, Frank t-norms that are NQD).

Contrarily, supermigrativity is preserved under ordinal sum constructions of semi-copulas, as the following result shows.

Proposition 2.4. Let $K$ be a finite or countable subset of $\mathbb{N}$. Let (]$a_{k}, b_{k}[)_{k \in K}$ be a family of nonempty, pairwise disjoint open subintervals of $\mathbb{I}$. Let $\left(S_{k}\right)_{k \in K}$ be a family of supermigrative semi-copulas. Then, the ordinal sum $S$ of $\left(S_{k}\right)_{k \in K}$ with respect to (]$a_{k}, b_{k}[)_{k \in K}$, denoted by $S=\left(\left\langle a_{k}, b_{k}, S_{k}\right\rangle\right)_{k \in K}$ and defined by

$$
S(x, y)= \begin{cases}a_{k}+\left(b_{k}-a_{k}\right) S_{k}\left(\frac{x-a_{k}}{b_{k}-a_{k}}, \frac{y-a_{k}}{b_{k}-a_{k}}\right), & (x, y) \in] a_{k}, b_{k}\left[^{2}\right. \\ \min \{x, y\}, & \text { elsewhere }\end{cases}
$$

is supermigrative.

Proof. First of all, it is well-known that an ordinal sum of semi-copulas is also a semicopula (see [16]). Hence, by Theorem 2.1, the claim reduces to showing that $S(\mathbf{x}) \geq S(\mathbf{y})$ 
whenever $\mathbf{x} \prec_{L} \mathbf{y}$. Recall that, for the 2-dimensional case, $\mathbf{x} \prec_{L} \mathbf{y}$ is equivalent to the two conditions $x_{1} \leq y_{1}$ and $\Pi_{2}(\mathbf{x})=\Pi_{2}(\mathbf{y})$.

First, suppose that both $\mathbf{x}$ and $\mathbf{y}$ belong to $] a_{k}, b_{k}\left[^{2}\right.$ for some $k \in K$. Set $\mathbf{x}^{k}:=\left(x_{1}^{k}, x_{2}^{k}\right)$ and $\mathbf{y}^{k}:=\left(y_{1}^{k}, y_{2}^{k}\right)$, where $x_{i}^{k}=\left(x_{i}-a_{k}\right) /\left(b_{k}-a_{k}\right)$ and, similarly, $y_{i}^{k}=\left(y_{i}-a_{k}\right) /\left(b_{k}-a_{k}\right)$ for $i=1,2$. It is immediate to see that $\mathbf{x}^{k}, \mathbf{y}^{k} \in \Delta_{2}$, with $x_{1}^{k} \leq y_{1}^{k}$. Moreover, since $\mathbf{x} \prec_{L} \mathbf{y}$, it is an elementary task to check that $x_{1}+x_{2} \leq y_{1}+y_{2}$. Thus, a simple algebraic calculation yields $\Pi_{2}\left(\mathbf{x}^{k}\right) \geq \Pi_{2}\left(\mathbf{y}^{k}\right)$. Consequently, being $S_{k}$ supermigrative, we can apply Lemma 2.3 to obtain $S_{k}\left(\mathbf{x}^{k}\right) \geq S_{k}\left(\mathbf{y}^{k}\right)$, which is equivalent to $S(\mathbf{x}) \geq S(\mathbf{y})$, so closing this first case. Here, notice that, since $y_{2} \leq x_{2} \leq x_{1} \leq y_{1}$, it is not possible that there exist $\left.\mathbf{x} \in\right] a_{k}, b_{k}\left[^{2}\right.$ and $\mathbf{y} \in] a_{j}, b_{j}\left[^{2}\right.$ for different indices $k, j \in K$.

Secondly, consider the case when $a_{k}<y_{2}$ and $x_{1}<b_{k} \leq y_{1}$ for some $k \in K$. Consequently, the claim easily becomes

$$
a_{k}+\left(b_{k}-a_{k}\right) S_{k}\left(\mathbf{x}^{k}\right) \geq y_{2}
$$

Since every supermigrative semi-copula is PQD and in view of inequality $\Pi_{2}\left(\mathbf{x}^{k}\right) \geq \Pi_{2}\left(\mathbf{y}^{k}\right)$, we obtain

$$
a_{k}+\left(b_{k}-a_{k}\right) S_{k}\left(\mathbf{x}^{k}\right) \geq a_{k}+\left(b_{k}-a_{k}\right) \Pi_{2}\left(\mathbf{y}^{k}\right) .
$$

Now, it can be seen by an elementary calculation that the assumptions of this second case ensure that the right-hand side of Eq. (3) dominates $y_{2}$ and, hence, in conclusion, Eq. (2) is shown.

Finally, if consider $y_{2} \leq a_{k}<x_{2}$ and $y_{1}<b_{k}$ for some $k \in K$, then the claim is a direct consequence of the fact that $S\left(\mathrm{x}^{k}\right)>a_{k}$. Moreover, in all the remaining cases, the condition $y_{2} \leq x_{2}$ ensures the claim, so the proof is completed.

In the framework of copulas, as known, the ordinal sum construction is a kind of mixture obtained from the initial family of copulas and some affine transformation of their arguments (see [17]). Roughly speaking, the previous result says that the supermigrativity of the initial copulas is preserved under this special mixing transformation. As a matter of fact, several notions of positive dependence for copulas (like positive quadrant dependence and stochastic increasingness) are also preserved under ordinal sum constructions. 
Curiously, Proposition 2.4 cannot be directly extended to the case of aggregation functions. Specifically, we can say that an aggregation function $A=\left(\left\langle a_{k}, b_{k}, A_{k}\right\rangle\right)_{k \in K}$ is an ordinal sum if it is defined as in Proposition 2.4 where a binary aggregation function $A_{k}$ replaces $S_{k}$ for every $k \in K$. In fact, in such a case, the property PQD is the key point for the preservation of supermigrativity, as stated by the following result.

Proposition 2.5. Let $A=\left(\left\langle a_{k}, b_{k}, A_{k}\right\rangle\right)_{k \in K}$ be an ordinal sum of supermigrative aggregation functions. Then, $A$ is supermigrative if, and only if, $A_{k}$ is $P Q D$ for every $k \in K$.

Proof. If $A_{k}$ is PQD for every $k \in K$, then the thesis follows by mimicking the proof of Proposition 2.4. Conversely, if $A$ is supermigrative, we assert that $A_{k}(1, y) \geq y$ for all $y \in \mathbb{I}$ and for any $k \in K$. Suppose ab absurdo there exists a $t \in] 0,1\left[\operatorname{such}\right.$ that $A_{k}(1, t)<t$ for some $k \in K$. Set $y_{t}:=a_{k}+\left(b_{k}-a_{k}\right) t$ and fix any $\left.x \in\right] a_{k}, b_{k}[$ : the supermigrativity of $A$ implies $A\left(x, y_{t}\right) \geq A\left(b_{k}, \frac{x y_{t}}{b_{k}}\right)$, or, equivalently,

$$
a_{k}+\left(b_{k}-a_{k}\right) A_{k}\left(\frac{x-a_{k}}{b_{k}-a_{k}}, t\right) \geq \frac{x y_{t}}{b_{k}} .
$$

By the monotonicity of $A_{k}$ in each argument, letting $x$ tend to $b_{k}$, the left hand-side of Eq. (4) tends to a finite limit dominated by $a_{k}+\left(b_{k}-a_{k}\right) A_{k}(1, t)$, which is, in its turn, strictly lower than $y_{t}$, as consequence of the fact that $A_{k}(1, t)<t$. Since the right handside of Eq. (4) trivially tends to $y_{t}$, we obtain the contradiction $y_{t}>y_{t}$, so showing the assertion. Owing to the supermigrativity of every summand, given any $(x, y) \in \mathbb{I}^{2}$ and any $k \in K$, we have that $A_{k}(x, y) \geq A_{k}(1, x y) \geq x y$, where the last inequality is due to the assertion.

This result should be compared with Proposition 2.4. Intuitively, it seems that a kind of "positive dependence" property should be required on the summands of an ordinal sum in order to guarantee that the overall construction preserves the supermigrative property.

\section{Supermigrativity in a multivariate setting}

In this section, we will denote by $A$ an arbitrary $d$-dimensional aggregation function ( $d$-aggregation function, for short), where $d$ is any natural number with $d \geq 2$. 
The characterizations of supermigrativity that have been formulated in Section 2 may give raise to different, alternative definitions of supermigrativity in high dimensions. In our view, a natural version of supermigrativity for $d$-aggregation functions coincides $d e$ facto with the notion of Schur geometrical concavity, as stated below.

Definition 3.1. A symmetric function $F: \mathbb{I}^{d} \rightarrow \mathbb{I}$ is called supermigrative if $F(\mathbf{x}) \geq F(\mathbf{y})$ whenever $\mathbf{x} \prec_{L} \mathbf{y}$.

Remark 3.1. Notice that $\mathbf{x} \prec_{L} \mathbf{y}$ implies the existence of a uniquely determined vector of real numbers $\left(\alpha_{0}, \alpha_{1}, \ldots, \alpha_{d}\right)$ belonging to $\left.] 0,1\right]^{d+1}$, with $\alpha_{0}=\alpha_{d}=1$, such that $x_{k}=\frac{\alpha_{k}}{\alpha_{k-1}} y_{k}$ for all $k=1, \ldots, d$.

By using this representation, we can easily derive the supermigrativity of the semicopula $M_{d}(\mathbf{x})=\min \left\{x_{1}, x_{2}, \ldots, x_{d}\right\}$ for every dimension $d$. In fact, $M_{d}(\mathbf{y})=y_{d}=\alpha_{d-1} x_{d} \leq$ $x_{d}=M_{d}(\mathbf{x})$. Accordingly, any function $F$ of the kind $F(\mathbf{x})=\Pi_{d}(\mathbf{x})^{\beta} \cdot M_{d}(\mathbf{x})^{\gamma}$ is supermigrative for any $\beta, \gamma>0$.

Obviously, according to Theorem 2.1, the above general notion of supermigrativity reduces to the one given in Definition 1.1 for $d=2$.

Here, we present two basic properties of supermigrative aggregation functions.

Lemma 3.1. Let $A$ be a supermigrative aggregation function. Then:

(a) 0 is an annihilator of $A$, i.e. $A(\mathbf{x})=0$ for all $\mathbf{x} \in \mathbb{I}^{d}$ such that $x_{j}=0$ for some $j \in\{1, \ldots, d\} ;$

(a) if $A$ has neutral element 1 , then $A(\mathbf{x}) \geq \Pi_{d}(\mathbf{x})$ for every $\mathbf{x} \in \mathbb{I}^{d}$.

Proof. The property of supermigrativity ensures that $A(\mathbf{x}) \geq A\left(1, \ldots, 1, \Pi_{d}(\mathbf{x})\right)$ for every $\mathbf{x} \in \mathbb{I}^{d}$. In particular, when all the components of $\mathbf{x}$ are equal to 0 , we immediately have that $A(1, \ldots, 1,0)=0$ and, hence, $A\left(u_{1}, \ldots, u_{d-1}, 0\right)=0$ for all $u_{1}, \ldots, u_{d-1} \in \mathbb{I}$, which entails the claim (a) by the symmetry of $A$. Moreover, part (b) follows by considering in previous inequality that, if 1 is a neutral element for $A$, then $A\left(1, \ldots, 1, \Pi_{d}(\mathbf{x})\right)=\Pi_{d}(\mathbf{x})$. 
Now, we address the problem of supermigrativity for two relevant families of symmetric $d$-aggregation functions, namely $d$-dimensional t-norms and quasi-arithmetic means.

In [12], the authors study the property of supermigrativity for continuous Archimedean t-norms. Associativity of these operations allows us to extend in a unique way any binary t-norm to a $d$-dimensional one (see, for instance, Definition 10.2 in [25]). Specifically, for a continuous and strictly decreasing function $f: \mathbb{I} \rightarrow[0, \infty]$, with $f(1)=0$, the explicit form of a continuous Archimedean $d$-dimensional t-norm is

$$
T(\mathbf{x})=f^{(-1)}\left(f\left(x_{1}\right)+\cdots+f\left(x_{d}\right)\right) \quad \text { for all } \mathbf{x} \in \mathbb{I}^{d} .
$$

Analogously to the classical case, Eq. (5) defines a strict- t-norm if $f(0)=+\infty$, otherwise the $t-$ norm is called nilpotent. We will see that the main properties regarding supermigrative continuous Archimedean t-norms maintain their validity passing from the bivariate to the multivariate case. From now on, it is intended that $T$ is a t-norm of arbitrary dimension $d \geq 2$, unless otherwise stated.

In [5], the conditions under which the additive generator $f$ of a strict, binary t-norm $T$ ensures that $T$ is supermigrative are given. We recall their result.

Proposition 3.2. Let $T$ be a binary continuous Archimedean t-norm generated by $f$. Then, $T$ is supermigrative if, and only if, $T$ is strict and $f^{-1}$ is log-convex.

In order to extend such a characterization to $d$-dimensions, we need a preliminary result.

Lemma 3.3. Let $f:[a, b] \rightarrow[0, \infty]$ be a strictly decreasing bijection, where $0 \leq a<b \leq 1$. Then, $f^{-1}$ is log-convex if, and only if,

$$
f(\alpha x)+f(y) \leq f(x)+f(\alpha y)
$$

for all $a \leq y \leq x \leq b$ and for all $\alpha \in \mathbb{I}$ such that $\alpha y \geq a$.

Proof. Due to a basic property of decreasing and convex real functions, $f^{-1}$ is log-convex if, and only if, the mapping $f_{\exp }:[\log a, \log b] \rightarrow[0, \infty]$ given by $f_{\exp }(t)=f(\exp (t))$ is 
convex. Then the claim is based upon the clear equivalence between Eq. (6) and

$$
f_{\exp }(t+h)-f_{\exp }(t) \geq f_{\exp }(w+h)-f_{\exp }(w),
$$

through the assignments $t:=\log (\alpha x), w:=\log (\alpha y)$ and $h:=-\log (\alpha)$.

Proposition 3.4. Let $T$ be a continuous Archimedean $t$-norm generated by $f$. Then, $T$ is supermigrative if, and only if, $T$ is strict and $f^{-1}$ is log-convex.

Proof. Suppose that $T$ is supermigrative. Then $T$ cannot be nilpotent since $T(x, \ldots, x) \geq$ $\Pi_{d}(x, \ldots, x)$ for every $x \in \mathbb{I}$ (Lemma 3.1, part (b)). In this case, by Lemma 3.3, it suffices to prove the validity of Eq. (6) applied to the additive generator $f$ for $a=0$ and $b=1$. It is quite easy to check that this task amounts to showing that the inequality $T(1, \ldots, 1, \alpha x, y) \geq T(1, \ldots, 1, x, \alpha y)$ holds true for all $\alpha \in \mathbb{I}$ and for all $(x, y) \in$ $\Delta_{2}$. Actually, this is assured by the supermigrativity of $T$, since $(1, \ldots, 1, \alpha x, y) \prec_{L}$ $(1, \ldots, 1, x, \alpha y)$, so closing the first part of the proof. Conversely, by Proposition 4.2 that will be presented below, we may limit ourselves to prove that $T\left(x_{1}, \ldots, x_{i-1}, \alpha x_{i}, \ldots, x_{d}\right) \geq$ $T\left(x_{1}, \ldots, x_{i}, \alpha x_{i+1}, \ldots, x_{d}\right)$ for all $\alpha \in \mathbb{I}$, for all $\mathbf{x} \in \Delta_{d}$ and for any index $i \in\{1, \ldots, d-1\}$. Again, it is quite easy to see that this task is equivalent to showing that $f\left(\alpha x_{i}\right)+f\left(x_{i+1}\right) \leq$ $f\left(x_{i}\right)+f\left(\alpha x_{i+1}\right)$, which is the same as Eq. (6) applied to the additive generator $f$, with $x_{i}$ and $x_{i+1}$ in place of $x$ and $y$, respectively, so definitely concluding the proof.

Since $\log$-convexity of a real function implies convexity, if $T$ is a binary continuous Archimedean t-norm that is supermigrative, then $T$ is a copula. However, in the multivariate case, convexity of the additive generator does not guarantee that the associated t-norm is a copula. Thus, there are continuous Archimedean and supermigrative $\mathrm{t}$-norms that are not copulas, as the following example shows.

Example 3.1. Let $T$ be the 3-dimensional strict t-norm generated by $f(t)=t^{-2}-t^{2}$ with $f^{-1}(u)=0.5 \sqrt{\left(u^{2}+1\right)^{0.5}-u / 2}$. As a consequence of [1, Example 4.4.8(b)], $T$ is not a copula, however $T$ is supermigrative since $f$ is log-convex. 
Now, we consider the case of quasi-arithmetic means (see, e.g., [22]) that, as known, are not semi-copulas. In the sequel, let $g: \mathbb{I} \rightarrow[c, d]$ be a strictly monotone bijection, where $[c, d] \subseteq[-\infty,+\infty]$. Moreover, the algebraic convention $-\infty+\infty$ equal to $-\infty$ or $+\infty$ is adopted, according to the increasing or decreasing monotonicity of $g$, respectively.

The $d$-aggregation function $M_{g}$ given by

$$
M_{g}(\mathbf{x})=g^{-1}\left(\frac{1}{n} \sum_{i=1}^{n} g\left(x_{i}\right)\right) \quad \text { for all } \mathbf{x} \in \mathbb{I}^{d}
$$

is called a quasi-arithmetic mean. Afterwards, we will call $g$ a generator of the quasiarithmetic mean $M_{g}$. It is well-known that if $g$ generates $M_{g}$, then also $g_{\beta, \gamma}$ generates $M_{g}$, where $g_{\beta, \gamma}:=\beta \cdot g+\gamma$, for any $\beta, \gamma \in \mathbb{R}$ such that $\beta \neq 0$. This allows us to assume for the rest of the section, without loss of generality, that any generator of a quasi-arithmetic mean is strictly decreasing and its range is exclusively one of the following:

(a) $c \in \mathbb{R} ; d=+\infty$;

(b) $c \in \mathbb{R} ; d \in \mathbb{R}$;

(c) $c=-\infty ; d=+\infty$;

(d) $c=-\infty ; d \in \mathbb{R}$.

Proposition 3.5. A quasi-arithmetic mean $M_{g}$ is supermigrative if, and only if, $g$ is in the case (a) and $g^{-1}$ is log-convex.

Proof. By Lemma 3.1, $M_{g}$ cannot be supermigrative in the cases (b) and (d), because in such cases $\mathbf{0}$ is not an annihilator. In case (c), given any $\alpha \in] 0,1[$, we immediately have that $\left(\alpha, \alpha^{2}, \ldots, \alpha^{2}\right) \prec_{L}\left(1, \alpha, \ldots, \alpha, \alpha^{d+1}\right)$. Therefore, if $M_{g}$ were supermigrative, we would find

$$
M_{g}\left(\alpha, \alpha^{2}, \ldots, \alpha^{2}\right) \geq M_{g}\left(1, \alpha, \ldots, \alpha, \alpha^{d+1}\right),
$$

or, equivalently,

$$
-\infty>(d-1) g\left(\alpha^{2}\right)+(3-d) g(\alpha)-g\left(\alpha^{d+1}\right) \geq g(1)=-\infty,
$$


which is a contradiction. Finally, in case (a), we can simply mimic the proof of the second part of Proposition 3.4.

\section{Supermigrativity and related inequalities}

Here, we present three inequalities, which coincide in the bivariate case with Eq. (1), clarifying their possible meaning and investigating their reciprocal relationships with Definition 3.1 in any dimension. From an application point of view, the proposed inequalities provide bounds for the aggregation process under a proportional rescaling of some of the input values, a property that could be particularly beneficial in image processing (see, e.g., $[7,8])$.

We denote by $\mathbf{x}_{\alpha, i}$ the vector that coincides with $\mathbf{x}$, except for its $i$-th component that is equal to $\alpha x_{i}$. Let $A$ be a symmetric $d$-aggregation function, $\mathbf{x} \in \Delta_{d}, \alpha \in \mathbb{I}$ and $i \in\{1, \ldots, d\}$. For such an $A$, we introduce the following three inequalities.

(I1) The first inequality states that, if we multiply the largest input value by a constant $\alpha$, then the output of the aggregation procedure driven by $A$ is larger than anyone obtained by multiplying any other input by the same $\alpha$. This statement is translated into the following inequality:

$$
A\left(\mathbf{x}_{\alpha, 1}\right) \geq \max _{i=2, \ldots, d} A\left(\mathbf{x}_{\alpha, i}\right)
$$

(I2) The second inequality states that, if we multiply the smallest input value by a constant $\alpha$, then the output of the aggregation procedure driven by $A$ is smaller than anyone obtained by multiplying any other input by the same $\alpha$. Formally, this entails

$$
\min _{i=1, \ldots, d-1} A\left(\mathbf{x}_{\alpha, i}\right) \geq A\left(\mathbf{x}_{\alpha, d}\right)
$$

(I3) The third inequality states that, if we multiply the $i$-th larger input value with a constant $\alpha$, then the output of the aggregation procedure driven by $A$ is larger than the one obtained by multiplying the subsequent input value with the same $\alpha$, for any $i \in\{1, \ldots, d-1\}$. It translates into the following form:

$$
A\left(\mathbf{x}_{\alpha, i}\right) \geq A\left(\mathbf{x}_{\alpha, i+1}\right) \quad \text { for } i=1, \ldots, d-1
$$


Remark 4.1. The inequality (9) was introduced for semi-copulas in [11, Definition 2.1]. In that work, given a vector of exchangeable lifetimes $\mathbf{X}=\left(X_{1}, \ldots, X_{d}\right)$, the aim was to find suitable multivariate notions of ageing that can extend the analogous properties well-studied in the univariate case. The idea, which dates back to the seminal paper [5], consists of expressing these properties via a suitable semi-copula (the so-called ageing function $B$ ), derived from a distortion of the survival copula of $\mathbf{X}$. It turned out that the Schur-concavity of the probability survival function $\bar{F}_{\mathbf{X}}$ of $\mathbf{X}$, which was recognized as a notion of multivariate IFR (i.e., increasing failure rate) already in [2, 3], could be equivalently expressed in terms of the supermigrativity of the associated aging function $B_{\bar{F} \mathbf{X}}$ in dimension $d=2$, as proved in [5], and, for any dimension $d$, in terms of inequality (9), as shown in [11].

In the sequel, we may omit the trivial cases $\alpha=0$ or $\alpha=1$.

Remark 4.2. Note that if $\mathrm{x} \in \Delta_{d}$, the vector $\mathrm{x}_{\alpha, i}$ does not generally belong to $\Delta_{d}$. The following rearrangement of $\mathbf{x}_{\alpha, i}$, denoted by $\mathbf{x}_{\alpha, i}^{\prime}$, belongs to $\Delta_{d}$ : set $s_{i}:=\max \{k \geq i$ : $\left.\alpha x_{i}<x_{k}\right\}$ and let $\mathbf{x}_{\alpha, i}^{\prime}$ coincide with $\mathbf{x}_{\alpha, i}$ if $s_{i}=i$. Otherwise, $\mathbf{x}_{\alpha, i}^{\prime}$ is obtained from $\mathbf{x}_{\alpha, i}$ by shifting its components $x_{i+1}, \ldots, x_{s_{i}}$ of one place to the left and moving the component $\alpha x_{i}$ to the $s_{i}$-th place.

Lemma 4.1. Let $\mathbf{x} \in \Delta_{d}$ and $\left.\alpha \in\right] 0,1\left[\right.$. Then, $\mathbf{x}_{\alpha, i}^{\prime} \prec_{L} \mathbf{x}_{\alpha, i+1}^{\prime}$ for any $i \in\{1, \ldots, d-1\}$.

Proof. Due to the previous remark, we only need to show that there exists an index $k_{i} \in$ $\{2, \ldots, d\}$ such that

$$
\Pi_{k}\left(T_{k}\left(\mathbf{x}_{\alpha, i}^{\prime}\right)\right) \leq \Pi_{k}\left(T_{k}\left(\mathbf{x}_{\alpha, i+1}^{\prime}\right)\right) \text { for all } k<k_{i}
$$

and

$$
\Pi_{k}\left(T_{k}\left(\mathbf{x}_{\alpha, i}^{\prime}\right)\right)=\Pi_{k}\left(T_{k}\left(\mathbf{x}_{\alpha, i+1}^{\prime}\right)\right) \quad \text { for all } k \geq k_{i} .
$$

Since $x_{i} \geq x_{i+1}$, it follows that $s_{i+1} \geq s_{i}$.

In the first case $s_{i+1}=s_{i}$, all the components of the two vectors $\mathbf{x}_{\alpha, i}^{\prime}$ and $\mathbf{x}_{\alpha, i+1}^{\prime}$ coincide, except for the $i$-th one and the $s_{i}$-th one, given by $x_{i+1}$ and $\alpha x_{i}$ for $\mathbf{x}_{\alpha, i}^{\prime}$, and by $x_{i}$ and 
$\alpha x_{i+1}$ for $\mathbf{x}_{\alpha, i+1}^{\prime}$. This implies that Eq. (10) boils down to $x_{i+1} \leq x_{i}$ for $k<s_{i}$, while Eq. (11) is trivially satisfied for $k \geq s_{i}$, so concluding the first case with $k_{i}=s_{i}$.

In the second case $s_{i+1} \geq s_{i}+1$, the only different components of the two vectors $\mathbf{x}_{\alpha, i}^{\prime}$ and $\mathbf{x}_{\alpha, i+1}^{\prime}$ are the $i$-th one and the ones between the $s_{i}$-th and the $s_{i+1}$-th place, given by $x_{i+1}$ and $\left(\alpha x_{i}, x_{s_{i}+1}, \ldots, x_{s_{i+1}-1}, x_{s_{i+1}}\right)$ for $\mathbf{x}_{\alpha, i}^{\prime}$, respectively, and by $x_{i}$ and $\left(x_{s_{i}+1}, x_{s_{i}+2}, \ldots, x_{s_{i+1}}, \alpha x_{i+1}\right)$ for $\mathbf{x}_{\alpha, i+1}^{\prime}$, respectively. Again, we immediately have that Eq. (10) holds true for $k<s_{i}$. Instead, for $k \in\left\{s_{i}, \ldots, s_{i+1}-1\right\}$, we derive that Eq. (10) reduces to $\alpha x_{i+1} \leq x_{k+1}$, which is assured by definition of $s_{i+1}$. Further, it is very easy to see that Eq. (11) is satisfied for $k \geq s_{i+1}$ and, hence, also the second case is concluded with $k_{i}=s_{i+1}$.

In the next result, we show that supermigrativity is actually equivalent to Eq. (9).

Proposition 4.2. Let $A$ be a symmetric d-aggregation function. Then, $A$ is supermigrative if, and only if, it satisfies Eq. (9).

Proof. Suppose first that $A$ is supermigrative. By the symmetry of $A$, Eq. (9) amounts to $A\left(\mathbf{x}_{\alpha, i}^{\prime}\right) \geq A\left(\mathbf{x}_{\alpha, i+1}^{\prime}\right)$, which holds true by Definition 3.1, seeing that $\mathbf{x}_{\alpha, i}^{\prime} \prec{ }_{L} \mathbf{x}_{\alpha, i+1}^{\prime}$ due to the previous lemma. Conversely, given any $\mathbf{x}, \mathbf{y}$ such that $\mathbf{x} \prec_{L} \mathbf{y}$, denote by $\mathbf{u}^{(k)}$ the vector given by $\left(y_{1}, \ldots, y_{k}, x_{k+1}, \ldots, x_{d}\right)$ for all $k=0, \ldots, d$ : note that $\mathbf{u}^{(0)}=\mathbf{x}$ and $\mathbf{u}^{(d)}=\mathbf{y}$. Owing to Remark 3.1, there exists a uniquely determined $(d+1)$-tuple of real numbers $\left(\alpha_{0}, \alpha_{1}, \ldots, \alpha_{d}\right)$ belonging to $\left.] 0,1\right]$, with $\alpha_{0}=\alpha_{d}=1$, such that $x_{k}=\frac{\alpha_{k}}{\alpha_{k-1}} y_{k}$ for all $k=1, \ldots, d$. Accordingly, it is not difficult to see that

$$
\mathbf{u}_{\alpha_{i}, i+1}^{(i)}=\mathbf{u}_{\alpha_{i+1}, i+1}^{(i+1)} \quad \text { for all } i=0, \ldots, d-1
$$

Particularly, the previous equation leads to $\mathbf{x}=\mathbf{u}_{\alpha_{0}, 1}^{(0)}=\mathbf{u}_{\alpha_{1}, 1}^{(1)}$ : applying Eq. (9) yields

$$
A(\mathbf{x})=A\left(\mathbf{u}_{\alpha_{1}, 1}^{(1)}\right) \geq A\left(\mathbf{u}_{\alpha_{1}, 2}^{(1)}\right)
$$

Repeating the above argument for a finite number of steps, we get

$$
A(\mathbf{x}) \geq A\left(\mathbf{u}_{\alpha_{1}, 2}^{(1)}\right) \geq A\left(\mathbf{u}_{\alpha_{2}, 2}^{(2)}\right) \geq \cdots \geq A\left(\mathbf{u}_{\alpha_{d}, d}^{(d)}\right)=A(\mathbf{y})
$$

so the proof is finished. 
Remark 4.3. According to the discussion in Remark 4.1, this latter result implies the following equivalence: $\bar{F}_{\mathbf{X}}$ is Schur-concave (i.e. multivariate IFR) if, and only if, the corresponding ageing function $B_{\bar{F}_{\mathbf{X}}}$ is Schur geometrically concave (i.e., supermigrative).

Since it is immediate to see that inequality (9) implies (7) and (8), by the previous result we immediately derive that, if $A$ is supermigrative, then it satisfies both (7) and (8). However, the converse implications are not true, as shown in the following examples.

Example 4.1. Let $S$ be the symmetric 3 -semi-copula given by $S(\mathbf{x})=x_{1} x_{3}$ for any $\mathbf{x} \in \Delta_{3}$. Note that Eq. (8) amounts to $S\left(\mathbf{x}_{\alpha, i}\right) \geq S\left(\mathbf{x}_{\alpha, 3}\right)$ for $i=1,2$, whose elementary proof is left to the reader, taking into account that $S\left(\mathbf{x}_{\alpha, 3}\right)=S\left(\mathbf{x}_{\alpha, 3}^{\prime}\right)=\alpha x_{1} x_{3}$. However, $S$ is not supermigrative, because if we consider, for instance, $\mathbf{x}=\left(\frac{1}{4}, \frac{1}{4}, \frac{1}{4}\right)$ and $\mathbf{y}=\left(\frac{1}{2}, \frac{1}{5}, \frac{5}{32}\right)$, one may easily check that $\mathbf{x} \prec_{L} \mathbf{y}$, but $S(\mathbf{x})<S(\mathbf{y})$.

Example 4.2. Let $B$ be the 3-aggregation function given by $B(\mathbf{x})=\Pi_{3}(\mathbf{x})^{5 / 3} \cdot M_{3}(\mathbf{x})$ : observe that $B$ is supermigrative, according to Remark 3.1. Let $A: \mathbb{I}^{3} \rightarrow \mathbb{I}$ be the symmetric function given, for $\mathbf{x} \in \Delta_{3}$, by

$$
A(\mathbf{x})= \begin{cases}x_{1} x_{2}^{3} x_{3}^{2}, & x_{1} x_{3}>x_{2}^{2} \\ B(\mathbf{x}), & x_{1} x_{3} \leq x_{2}^{2}\end{cases}
$$

We leave to the reader the relatively simple, but somewhat tedious, task of showing that $A$ is a continuous aggregation function. Note that Eq. (7) amounts to

$$
A\left(\mathbf{x}_{\alpha, 1}\right) \geq A\left(\mathbf{x}_{\alpha, i}\right) \quad \text { for } i=2,3
$$

Being $A$ continuous, we may limit ourselves to $\mathbf{x} \in \Delta_{3}$ such that $1>x_{1}>x_{2}>x_{3}>0$. We emphasize that we shall not examine the cases when Eq. (12) goes back to $B\left(\mathbf{x}_{\alpha, 1}\right) \geq B\left(\mathbf{x}_{\alpha, i}\right)$ as consequence of the supermigrativity of $B$, seeing that $\mathbf{x}_{\alpha, 1} \prec_{L} \mathbf{x}_{\alpha, i}$ for $i=2$, 3 . Let us start with Eq. (12) for $i=3$, which presents a total of three cases each of which composed by only one non-trivial subcase. In the first case $\alpha x_{1} \geq x_{2}$, it is enough to consider the subcase $\alpha x_{1} x_{3}>x_{2}^{2}$ : under these assumptions, Eq. (12) is equivalent to $\alpha \leq 1$. In the 
second case, given by $x_{2}>\alpha x_{1} \geq x_{3}$, we immediately get $\alpha x_{1} x_{3}<x_{2} x_{3}<x_{2}^{2}$, hence we may restrict our study to the subcase $x_{2} x_{3}>\left(\alpha x_{1}\right)^{2}$ : it is very easy now to see that Eq. (12) amounts to $\alpha x_{1}^{4} \geq\left(x_{2} x_{3}\right)^{2}$, which is clearly implied by $\alpha x_{1} \geq x_{3}$ and $x_{1}>x_{2}$.

In the third case $x_{3}>\alpha x_{1}$, being again $\alpha x_{1} x_{3}<x_{2}^{2}$, it suffices to consider the subcase $\alpha x_{1} x_{2}>x_{3}^{2}$, when Eq. (12) boils down to $x_{1} x_{3} \geq\left(\alpha x_{2}\right)^{2}$, which is evidently entailed by $\alpha x_{2}<\alpha x_{1}<x_{3}$, so definitely closing the proof of Eq. (12) for $i=3$.

Now, let us examine Eq. (12) for $i=2$, which presents a total of five cases each of which composed by two non-trivial subcases, except for the fifth one. In the first case $\alpha x_{1} \geq x_{2}$ and $\alpha x_{2} \geq x_{3}$, the first subcase is given by $\alpha x_{1} x_{3}>x_{2}^{2}$ and $x_{1} x_{3}>\left(\alpha x_{2}\right)^{2}$. Under these assumptions, Eq. (12) is equivalent to the trivial condition $\alpha^{2} \leq 1$. Notice that if $x_{1} x_{3} \leq\left(\alpha x_{2}\right)^{2}$, then we directly get $\alpha x_{1} x_{3}<x_{1} x_{3} \leq\left(\alpha x_{2}\right)^{2}<x_{2}^{2}$, hence the second subcase is given by $\alpha x_{1} x_{3} \leq x_{2}^{2}$ and $x_{1} x_{3}>\left(\alpha x_{2}\right)^{2}$ : it is not difficult now to show that Eq. (12) amounts to the assumption $x_{1} x_{3}>\left(\alpha x_{2}\right)^{2}$. In the second case $\alpha x_{1} \geq x_{2}$ and $\alpha x_{2}<x_{3}$, observe that $\alpha x_{1} x_{2} \geq x_{2}^{2}>x_{3}^{2}$, thus the first relevant subcase is given by $\alpha x_{1} x_{3}>x_{2}^{2}$, when Eq. (12) becomes $x_{2} \geq \alpha x_{3}$, which obviously holds true. In the second subcase, given by $\alpha x_{1} x_{3} \leq x_{2}^{2}$, Eq. (12) leads to $\alpha x_{2} x_{3} \leq x_{1}^{2}$, which is ensured by the fact that $\alpha x_{2}<x_{3}<x_{1}$. In the third case $x_{2}>\alpha x_{1} \geq x_{3}$ and $\alpha x_{2} \geq x_{3}$, the first subcase is given by $x_{2} x_{3}>\left(\alpha x_{1}\right)^{2}$ and $x_{1} x_{3}>\left(\alpha x_{2}\right)^{2}$, when Eq. (12) leads to $x_{1}>x_{2}$. Notice that if $x_{1} x_{3} \leq\left(\alpha x_{2}\right)^{2}$, then $x_{2} x_{3}<x_{1} x_{3} \leq\left(\alpha x_{2}\right)^{2}<\left(\alpha x_{1}\right)^{2}$, hence the second subcase is $x_{1} x_{3}>\left(\alpha x_{2}\right)^{2}$ and $x_{2} x_{3} \leq\left(\alpha x_{1}\right)^{2}$, when Eq. (12) is exactly equivalent to the assumption $x_{1} x_{3}>\left(\alpha x_{2}\right)^{2}$. In the fourth case $x_{2}>\alpha x_{1} \geq x_{3}$ and $\alpha x_{2}<x_{3}$, notice that $\alpha x_{1} x_{2} \geq x_{3} x_{2}>x_{3}^{2}$, hence the first subcase is given by $x_{2} x_{3}>\left(\alpha x_{1}\right)^{2}$, when Eq. (12) boils down to $\alpha x_{1}^{2} \geq x_{2} x_{3}$, which is implied by $\alpha x_{1} \geq x_{3}$ and $x_{1}>x_{2}$. In the second relevant subcase, given by $x_{2} x_{3} \leq\left(\alpha x_{1}\right)^{2}$, Eq. (12) goes to $x_{1}^{2} \geq \alpha x_{2} x_{3}$, which is clearly entailed by $x_{1}>x_{3}>\alpha x_{2}$. Finally, in the fifth and last case $\alpha x_{1}<x_{3}$, it suffices to analyze the subcase $\alpha x_{1} x_{2}>x_{3}^{2}$, when Eq. (12) leads to $x_{1}>x_{2}$, so definitely concluding the proof that $A$ satisfies Eq. (7).

However, $A$ is not supermigrative, because if we consider, for instance, $\mathbf{x}=\left(\frac{9}{10}, \frac{3}{5}, \frac{3}{5}\right)$ and $\mathbf{y}=\left(1, \frac{27}{40}, \frac{12}{25}\right)$, one may easily check that $\mathbf{x} \prec_{L} \mathbf{y}$, but $A(\mathbf{x})<A(\mathbf{y})$. 
The relationship between the three inequalities formulated in (7), (8) and (9) may be addressed also under a different point of view. Given any symmetric $d$-aggregation function $A$, consider the binary aggregation function induced by $A$, denoted with $A_{12}$, and defined as

$$
A_{12}(x, y)=A(1, \ldots, 1, x, y) .
$$

According to Definition 1.1, $A_{12}$ is supermigrative if, and only if, it satisfies Eq. (1). In this setting, Eq. (1) can be easily reframed as follows: given any $\alpha \in \mathbb{I}$ and any $(x, y) \in \Delta_{2}$

$$
A\left(\mathbf{v}_{\alpha, d-1}\right) \geq A\left(\mathbf{v}_{\alpha, d}\right)
$$

where $\mathbf{v}:=(1, \ldots, 1, x, y)$. Thus, it can be easily show that, if $A$ satisfies Eq. (8) (respectively, Eq. (9)), then it fulfills Eq. (13) as well and, hence, it is supermigrative. In other words, the supermigrativity of $A$ entails the supermigrativity of $A_{12}$, but, oddly enough, the same implication holds assuming Eq. (8) rather than the stronger condition given by Eq. (9). On the contrary, if $A$ fulfills Eq. (7), then $A_{12}$ need not be supermigrative, as the following example shows.

Example 4.3. Consider the 3-aggregation function $A$ illustrated in Example 4.2. It is not difficult to see that the induced function $A_{12}$ is given, for $(x, y) \in \Delta_{2}$, by

$$
A_{12}(x, y)= \begin{cases}x^{3} y^{2}, & y>x^{2} ; \\ x^{5 / 3} y^{8 / 3}, & y \leq x^{2} .\end{cases}
$$

As shown in Example 4.2, $A$ verifies Eq. (7), but the above described $A_{12}$ is not supermigrative, because, for instance, Eq. (1) fails for $x=3 / 5, y=1 / 2$ and $\alpha=5 / 6$.

Finally, notice that, if $A$ is a $d$-dimensional symmetric aggregation function such that $A_{12}$ is supermigrative, then $A$ need not be supermigrative, as the following example shows. Example 4.4. Consider the following symmetric 3-aggregation function given, for $\mathrm{x} \in \Delta_{3}$, by

$$
A(\mathbf{x})= \begin{cases}\Pi_{3}(\mathbf{x}), & \text { if } x_{1}<1 \\ \sqrt{\Pi_{3}(\mathbf{x})}, & \text { otherwise }\end{cases}
$$


It is immediate to see that $A_{12}(\alpha x, y)=A_{12}(x, \alpha y)=\sqrt{\alpha x y}$, for any $\alpha \in \mathbb{I}$ and for all $x, y \in \mathbb{I}$. However, $A$ is not supermigrative, because if we consider, for instance, $\mathbf{x}=\left(\frac{1}{2}, \frac{1}{2}, \frac{1}{4}\right)$ and $\mathbf{y}=\left(1,1, \frac{1}{16}\right)$, one may easily check that $\mathbf{x} \prec_{L} \mathbf{y}$, but $A(\mathbf{x})<A(\mathbf{y})$.

\section{Conclusions}

We have revisited the notion of bivariate supermigrativity for semi-copulas and extended it to the case of aggregation functions in higher dimensions. In particular, we have shown how supermigrativity is related to logarithmic majorization of real vectors. Various alternative characterizations are illustrated, together with some possible weaker versions of supermigrativity. Relationships with notions of multivariate aging are emphasized.

\section{Acknowledgements}

We would like to thank the anonymous Reviewers for their careful reading and detailed comments that have been helpful for providing a better version of the present work.

The first author was partially supported by the Free University of Bozen-Bolzano, Faculty of Economics and Management, via the project AIDA.

[1] C. Alsina, M. J. Frank, and B. Schweizer. Associative functions. Triangular norms and copulas. World Scientific Publishing Co. Pte. Ltd., Hackensack, NJ, 2006.

[2] R. E. Barlow and M. B. Mendel. De Finetti-type representations for life distributions. J. Amer. Statist. Assoc., 87(420):1116-1122, 1992.

[3] R. E. Barlow and F. Spizzichino. Schur-concave survival functions and survival analysis. J. Comput. Appl. Math., 46(3):437-447, 1993.

[4] B. Bassan and F. Spizzichino. Dependence and multivariate aging: the role of level sets of the survival function. In Y. Hayakawa, T. Irony, and M. Xie, editors, System and Bayesian reliability, volume 5 of Series on Quality, Reliability and Engineering Statistics, pages 229-242. World Scientific Publishing, Singapore, 2001.

[5] B. Bassan and F. Spizzichino. Relations among univariate aging, bivariate aging and dependence for exchangeable lifetimes. J. Multivariate Anal., 93(2):313-339, 2005. 
[6] F. Belzunce, C. Martínez-Riquelme, F. Pellerey, and S. Zalzadeh. Comparison of hazard rates for dependent random variables. Statistics, in press, 2015.

[7] H. Bustince, B. De Baets, J. Fernandez, R. Mesiar, and J. Montero. A generalization of the migrativity property of aggregation functions. Inform. Sci., 191:76-85, 2012.

[8] H. Bustince, J. Fernandez, R. Mesiar, J. Montero, and R. Orduna. Overlap functions. Nonlinear Anal., 72(3-4):1488-1499, 2010.

[9] H. Bustince, J. Montero, and R. Mesiar. Migrativity of aggregation functions. Fuzzy Sets and Systems, 160(6):766-777, 2009.

[10] R. Cerqueti and F. Spizzichino. Extension of dependence properties to semi-copulas and applications to the mean-variance model. Fuzzy Sets and Systems, 220:99-108, 2013.

[11] F. Durante, R. Foschi, and F. Spizzichino. Ageing function and multivariate notions of NBU and IFR. Probab. Engrg. Inform. Sci., 24(2):263-278, 2010.

[12] F. Durante and R. Ghiselli-Ricci. Supermigrative semi-copulas and triangular norms. Inform. Sci., 179(15):2689-2694, 2009.

[13] F. Durante and R. Ghiselli-Ricci. Supermigrative copulas and positive dependence. AStA Adv. Stat. Anal., 96(3):327-342, 2012.

[14] F. Durante, J. J. Quesada-Molina, and C. Sempi. Semicopulas: characterizations and applicability. Kybernetika (Prague), 42(3):287-302, 2006.

[15] F. Durante and P. Sarkoci. A note on the convex combinations of triangular norms. Fuzzy Sets and Systems, 159(1):77-80, 2008.

[16] F. Durante and C. Sempi. Semicopulæ. Kybernetika (Prague), 41(3):315-328, 2005.

[17] F. Durante and C. Sempi. Principles of Copula Theory. CRC/Chapman \& Hall, Boca Raton, FL, 2016.

[18] J. Fodor and I. J. Rudas. On continuous triangular norms that are migrative. Fuzzy Sets and Systems, 158(15):1692-1697, 2007.

[19] J. Fodor and I. J. Rudas. Migrative t-norms with respect to continuous ordinal sums. Inform. Sci., In Press, 2011. 
[20] R. Foschi. Hyper-dependence, hyper-ageing properties and analogies between them: a semigroupbased approach. Kybernetika (Prague), 49(1):96-113, 2013.

[21] R. Foschi and F. Spizzichino. Semigroups of semicopulas and evolution of dependence at increase of age. Mathware 83 Soft Computing, XV(1):95-111, 2008.

[22] M. Grabisch, J.-L. Marichal, R. Mesiar, and E. Pap. Aggregation Functions. Encyclopedia of Mathematics and its Applications (No. 127). Cambridge University Press, New York, 2009.

[23] K. Guan. Some properties of a class of symmetric functions. J. Math. Anal. Appl., 336(1):70-80, 2007.

[24] H. Joe. Dependence Modeling with Copulas. Chapman \& Hall/CRC, London, 2014.

[25] E. P. Klement, R. Mesiar, and E. Pap. Triangular norms, volume 8 of Trends in Logic-Studia Logica Library. Kluwer Academic Publishers, Dordrecht, 2000.

[26] E. P. Klement, R. Mesiar, and E. Pap. A universal integral as common frame for Choquet and Sugeno integral. IEEE Trans. Fuzzy Systems, 18(1):178-187, 2010.

[27] E. P. Klement, R. Mesiar, F. Spizzichino, and A. Stupňanová. Universal integrals based on copulas. Fuzzy Optim. Decis. Mak., 13(3):273-286, 2014.

[28] C.-D. Lai and M. Xie. Stochastic ageing and dependence for reliability. Springer, New York, 2006.

[29] J.-F. Mai and M. Scherer. Simulating copulas. Imperial College Press, London, 2012.

[30] A. W. Marshall, I. Olkin, and B. C. Arnold. Inequalities: theory of majorization and its applications. Springer Series in Statistics. Springer, New York, second edition, 2011.

[31] R. B. Nelsen. An Introduction to Copulas. Springer Series in Statistics. Springer, New York, second edition, 2006.

[32] F. Pellerey and F. Spizzichino. Joint weak hazard rate order under non-symmetric copulas. Depend. Model., 4(1):190-204, 2016.

[33] H.-N. Shi and J. Zhang. Compositions involving Schur geometrically convex functions. J. Inequal. Appl., 2015(1):1-9, 2015.

[34] F. Spizzichino. Subjective probability models for lifetimes, volume 91 of Monographs on Statistics and Applied Probability. Chapman \& Hall/CRC, Boca Raton, FL, 2001. 
[35] F. Spizzichino. Semi-copulas and interpretations of coincidences between stochastic dependence and ageing. In P. Jaworski, F. Durante, W. Härdle, and T. Rychlik, editors, Copula Theory and its Applications, volume 198 of Lecture Notes in Statistics - Proceedings, pages 237-254. Springer, Berlin Heidelberg, 2010.

[36] S. Zalzadeh and F. Pellerey. A positive dependence notion based on componentwise unimodality of copulas. Statist. Probab. Lett., 112:51-57, 2016. 